\title{
La regulación emocional e impulsividad y su relación con el juego patológico
}

\author{
Jessica Muñoz Mendiola \\ al267600@uji.es \\ Jessica Gregori Coronado \\ al202166@uji.es \\ JULIO ABAD \\ julioabad@hotmail.es \\ JUANA BRETÓN-LÓPEZ \\ breton@psb.uji.es
}

\section{Resumen}

Introducción: El juego patológico implica un comportamiento problemático, persistente y recurrente de juego con importantes pérdidas de dinero, que expone a la persona a consecuencias negativas en el plano social, profesional e individual. En el juego patológico existen una serie de factores implicados como factores predisponentes y variables relacionadas. El presente trabajo analiza la relación de las variables de regulación emocional e impulsividad con los trastornos del juego. Método: Se llevó a cabo un estudio exploratorio con 12 pacientes que pertenecían a la asociación PATIM (Asociación para la Intervención e Integración en Adicciones y Otras Conductas). Todos los participantes complementaron un cuestionario de screening de adicción al juego, la escala de dificultades en la regulación emocional (DERS) y la escala de impulsividad de Barratt (BISS-11). Resultados: Se observaron puntuaciones por encima de la puntuación normativa en la variable de regulación emocional, tanto en la puntuación total como en las subescalas. Sin embargo, en la impulsividad, las puntuaciones se encontraban por debajo de la puntuación normativa. Por último, los resultados del focus group muestran la repercusión de las emociones en dicha adicción comportamental. Conclusiones: El presente estudio revela que las dificultades en la regulación emocional tienen una importancia en el juego patológico. Por el contrario, en la impulsividad, no se encuentran medidas superiores a la puntuación de corte en población normal en la muestra de juego patológico del estudio. Finalmente, la relevancia del manejo de las emociones nos sugiere la importancia del desarrollo de estrategias de regulación emocional como propuesta de tratamiento.

Palabras clave: juego patológico, factores predisponentes, variables relacionadas, regulación emocional e impulsividad. 


\section{Abstract}

Introduction: Gambling disorder implies a problematic, persistent and recurrent gaming behaviour, and significant losses of money, which expose a person to negative consequences in social, professional and individual terms. A series of elements involved as predisposed factors and related variables exist in gambling disorders. This study analysed the emotional regulation and impulsivity variables related to gambling behaviour. Method: An exploratory study with 12 patients who belong to the PATIM Association (Association for Intervening and Integrating Addictions and Other Behaviours) was carried out. The participants completed a questionnaire for screening addiction to gaming, the Difficulties in Emotion Regulation Scale (DERS) and the Barratt Impulsiveness Scale (BISS-11). Results: Scores went above the normative score for the emotional regulation variable for both the total score and subscales. However for impulsiveness, scores were below the standard score. The Focus Group results showed the impact of emotions on such behaviour. Conclusions: The present study reveals that emotional regulation difficulties are very important in pathological gambling. Conversely with impulsivity, no scores were obtained above the cut-off score measure in the normal population in the study pathological gambling sample. In short, the relevance of handling emotions suggests the importance of developing emotional regulation as a proposal of treatment strategies.

Keywords: Pathological gambling, predisposed factor, related variables, emotion regulation and impulsivity.

\section{Introducción}

El concepto referente a la relación excesiva del ser humano con el juego ha ido evolucionando y modificándose a lo largo de diferentes etapas. Históricamente, el concepto juego era considerado una debilidad moral, propia de personas con poca voluntad o viciosas, sin tener en cuenta el sufrimiento que provoca dicha conducta. (Dóminguez, 2007 a). En la primera mitad del siglo $\mathrm{XX}$, se dieron las primeras explicaciones, desde un marco psicoanalítico, sobre el juego excesivo (Freud, 1948).

En 1975, según Bergel, comenzó a entenderse como una enfermedad psicológica, concretamente, como una manifestación de una neurosis subyacente. Hasta 1975 no se comenzó a estudiar la ludopatía como enfermedad y fue en 1979 cuando Morán la definió como juego patológico. Su reconocimiento oficial se produce en 1980, cuando la American Psychiatric Association, en el Manual Diagnóstico y Estadístico de los Trastornos Mentales (DSM) lo incluye en una de sus categorías.

Actualmente, el DSM-V (American Psychiatric Association, 2013) ha introducido modificaciones importantes que han supuesto un cambio en la consideración psicopatológica de este trastorno. En cualquier caso, se entiende juego patológico como un comportamiento problemático, persistente y recurrente, que provoca un deterioro o malestar clínicamente significativo. Está caracterizado por la dificultad o imposibilidad de resistir el impulso, el deseo o la tentación de realizar algún acto que resulta nocivo para el paciente o para las personas que lo rodean (conducta de juego). 
Los estudios epidemiológicos han ido contemplando un aumento de la patología del juego. Los expertos como Bowden y Clark, en el 2011, apuntan que el incremento de juegos legalizados junto con la facilidad de juego y la publicidad han influenciado en la incidencia de dicho trastorno. Este acrecentamiento afecta a la población en general. Sin embargo, hay una elevada incidencia en la población joven (Griffiths y Wood, 2000). Los porcentajes de prevalencia para este colectivo son altos, situándose entre el $3 \%$ y el $18 \%$ (Esteve y cols., 2014). En cuanto al porcentaje total, se ha llegado a un consenso en el cual se habla una estimación de un 2-3 \% de prevalencia del trastorno en la población (APA, 2005) y, dentro de este porcentaje, un $40 \%$ de los casos fluctúa entre los 18-30 años (Chóliz, 2006).

A partir de ciertos estudios epidemiológicos pioneros, surge un perfil con características descriptivas de los jugadores patológicos (Becoña, 1993; Jiménez y Fernández de Haro, 1999). Dichas características sociodemográficas se mantienen comunes en estudios posteriores (Domínguez, 2007 b y 2009). Además, hay dos hombres por cada mujer jugadora patológica, por lo que se aprecia una prevalencia del género masculino (Wardle y cols., 2011). Predominan los jugadores jóvenes (como hemos mencionado, el $40 \%$ de todos tienen entre 18 y 30 años). Tienen un menor nivel académico, menos ingresos económicos, su ocupación laborar es similar a la de la población no jugadora (no existe asociación entre mayor nivel de juego y una determinada profesión) y las máquinas tragaperras aparecen como juego predominante (en el $75 \%$ de los casos) (Williams y cols., 2012). Los cambios observados en relación al juego (su legalización y proliferación) han causado que los juegos de azar, actualmente, estén al alcance de cualquier persona, lo que puede propiciar que el perfil tradicional del jugador cambie (Candiani, 2012), más aún con el juego online.

La adicción al juego y sus repercusiones causan un incremento en la sintomatología del jugador (Caballo, Salazar y Carrobles, 2014). Desde una perspectiva psicopatológica, la comorbilidad de enfermedades mentales con el juego patológico es destacable. Los estudios sobre comorbilidad del juego patológico con trastornos del eje I registran la existencia de altas tasas de trastornos del estado de ánimo como la depresión mayor, distimia, manía e hipomanía (que oscilan entre el 33 y $76 \%$ ), trastornos de ansiedad (9-40\%) o trastorno por déficit de atención con hiperactividad (20\%) (Jiménez-Murcia y cols., 2009). Cabe agregar, en cuanto al eje tipo II, que se ha examinado la presencia de patologías asociadas al juego patológico, estipulando que los trastornos más comunes son los del clúster B del DSM-IV-TR, que coinciden con los sujetos inestables emocionalmente (Fernández y Echeburúa, 2006). Profundizando en los diferentes trastornos de la personalidad, se incluye el obsesivo compulsivo (entre el 27,3 y el $64 \%$ de los jugadores), el trastorno límite (62\%), el narcisista (23,6 y $53 \%$ ), el antisocial (entre el 23,6 \% y el $35 \%$ ) y el dependiente (entre el 29,7 \%) (Odlaug y cols., 2013). Además, la tasa de prevalencia de un consumo excesivo de alcohol y otras drogas entre los jugadores patológicos es muy alta, alrededor de un 11-70 \% de los casos (French, Maclean y Etner, 2008).

La falta de control de los impulsos ha sido una característica vinculada al perfil psicológico de los pacientes con problemas de juego (Bottesi y Ghis, 2014). Se ha comprobado que la gravedad de la sintomatología del juego patológico estaba relacionada con los rasgos de impulsividad y compulsividad ya que la disminución de estos rasgos en escalas de impulsividad estaba vinculada con la disminución de los síntomas del juego patológico (Blanco y cols., 2009). Existe una mayor prevalencia de rasgos como impulsividad y búsqueda de sensaciones en los jugadores patológicos.

Podemos ver alrededor de un 35-43\% de comorbilidad entre la adicción al juego y el control de los impulsos (Jiménez-Murcia y cols., 2009). Los resultados muestran la importancia de la impulsividad en los jugadores patológicos (Fernández y Echeburúa, 2006). En concordancia a la comorbilidad entre la falta de control de impulsos y el juego patológico podemos 
decir que la impulsividad es uno de los factores predisponentes o variables relacionados con esta adicción comportamental.

Las conductas impulsivas de juego patológico se han puesto en relación a su vez con cuestiones de regulación emocional (Esteve y cols., 2014). Las dificultades de la regulación emocional están relacionadas con las dificultades de control emocional (Gratz y Roemer, 2004) y se ha puesto de manifiesto que las personas con problemas de juego utilizan esta conducta como primera estrategia de regulación emocional (Korman y cols., 2008). Se puede apreciar una relación entre el juego y la expectativa de conseguir estados anímicos positivos o aliviar estados anímicos negativos (Shead, Callan y Hodgins, 2008) y se han observado dificultades en la regulación emocional en personas adultas que están en tratamiento por problemas de juego patológico (Williams y cols., 2012).

El objetivo de este trabajo es estudiar las variables de regulación emocional e impulsividad en el trastorno de juego en una muestra de pacientes de juego patológico.

\section{Método}

\section{Participantes}

La muestra está compuesta por un conjunto de pacientes que presentaban diagnóstico de juego patológico (excepto un paciente en remisión) y que acuden a la asociación PATIM (Asociación para la Intervención e Integración en Adicciones y Otras Conductas) de Castellón y Valencia. Se compone de un total de 12 varones, con edades comprendidas entre 19 y 69. La edad media de la muestra es de 38,64 y su desviación típica es 15,57.

Respecto a los datos sociodemográficos de estos pacientes, podemos apreciar en cuanto a su situación civil que un $36,36 \%$ es soltero, un $27,27 \%$ tiene pareja, un $18,18 \%$ está casado y un 18,18 \% está divorciado. En relación a la situación laboral, un 27,27 \% es estudiante, un $36,36 \%$ es activo, un $9,09 \%$ es desempleado, un $9,09 \%$ es prejubilado y un $18,18 \%$ es jubilado.

\section{Instrumentos}

Escala NODS (NORC DSM-IV Screen for Gambling Problem) (Gerstein y cols.,1999; versión española, Becoña, 2004). Esta escala evalúa el cumplimiento de criterios diagnósticos y el grado de gravedad del trastorno de juego patológico a lo largo de la vida y el último año. Consta de 17 ítems agrupados en las diez categorías del DSM-IV para el diagnóstico de juego patológico (5 o más criterios). Diferencia la tipología del jugador: patológico (5 o más criterios), problema (3 o 4 criterios) y en riesgo (1 o 2 criterios).

Escala de dificultades en la regulación emocional (DER; Gratz y Roemer, 2004). La escala de dificultades en la regulación emocional evalúa diferentes componentes desadaptativos de la regulación emocional (Gratz y Roemer, 2004). La adaptación al castellano (Hervás y Jodar, 2008) cuenta con una reducción de factores e ítems con respecto a la original. La escala consta de 28 ítems, mientras que la inicial mostraba 36 . El formato de respuesta es de escala Likert de uno a cinco ( 1 = casi nunca y 5 = casi siempre) y el sujeto selecciona en función de la frecuencia con la que se sienta identificado en cada afirmación. La versión española está compuesta por cinco factores: desatención emocional, confusión emocional, rechazo emocional, interferencia emocional y descontrol emocional. Las diferentes escalas presentan 
una elevada consistencia interna (valores a comprendidos entre 0,8 y 0,89) así como también la escala total $(\alpha=0,93)$.

Escala de impulsividad de Barrat (BIS-11; Oquendo y cols., 2001). La escala de impulsividad de Barrat, versión 11 (BIS-11) evalúa impulsividad. Está compuesta por 30 ítems distribuidos en tres subescalas: impulsividad cognitiva (atención), impulsividad motora e impulsividad no planeada. Cada ítem consta de cinco opciones de respuesta con formato Likert de cero a cuatro ( 0 = raramente o nunca; 1 = ocasionalmente; 3 = a menudo; $4=$ siempre o casi siempre $)$ y el sujeto deberá seleccionar en función de la frecuencia con la que realice cada afirmación. La escala muestra unas propiedades psicométricas aptas, con un coeficiente alfa de 0,75. La consistencia interna es elevada, alrededor de 0,8. La fiabilidad test-retest es de 0,89.

Además de la administración de los instrumentos anteriormente mencionados, se llevó a cabo un focus group aproximadamente de 30 minutos de duración, con el objetivo de recoger una mayor información que permitiera conocer de manera exhaustiva las variables relacionadas con la regulación emocional. El instrumento del focus group consistió en citar a los pacientes en pequeños grupos, concretamente en tres grupos de cuatro personas, en una sala de la misma asociación para contestar, desde la experiencia personal, a distintos aspectos específicos asociados a la regulación de las emociones. Esta técnica elaborada para la investigación estaba compuesta de seis preguntas relacionadas con distintos aspectos específicos de la regulación emocional.

\section{Diseño}

La presente investigación es un trabajo empírico con un diseño exploratorio (León y Montero, 2007).

\section{Procedimiento}

El procedimiento mediante el cual llevábamos a cabo nuestro estudio constó de varios pasos. En primer lugar, se seleccionaron los centros donde cabía la posibilidad de obtener una muestra de jugadores patológicos y se contactó con el psicólogo responsable de cada centro para explicarle las características del estudio y obtener su autorización. Se contactó con la asociación PATIM que disponía de pacientes con dicha patología y aceptó la colaboración al estudio. En segundo lugar, se llevó a cabo una reunión con el psicólogo y el trabajador social de PATIM en la que se seleccionó una muestra de pacientes que acudían a la asociación de Valencia o de Castellón para la terapia psicológica en rehabilitación e inserción social. Los criterios de selección fueron los siguientes: ser mayor de edad, aceptar de forma voluntaria y expresa la participación en el estudio y presentar o haber presentado un diagnóstico de juego patológico, de acuerdo con los criterios específicos en el DSM-V. En tercer lugar, para los pacientes que habían aceptado con antelación participar en el estudio, tras explicarles las instrucciones y en qué consistiría, respetando la fecha y el horario que habíamos acordado con el psicólogo, los agrupamos en tres grupos concretando el día. La evaluación se llevó a cabo en una sala proporcionada por la misma asociación; al primer grupo lo citamos en la asociación de Castellón y al segundo y al tercero en la asociación de Valencia. Durante la evaluación se hizo una presentación del equipo de investigación y se les explicó, de nuevo, la naturaleza del estudio, enfatizando el anonimato y la confidencialidad, tanto de los cuestionarios que iban a rellenar como de sus respuestas a las preguntas del focus group. De la misma forma, se les informó que, si en algún momento deseaban abandonar el estudio, podrían hacerlo. En la 
ejecución del focus group, una psicóloga estudiante del MPGS (Máster en Psicología General Sanitaria) actuaba de investigadora y moderadora, realizando las preguntas oportunas y facilitando las discusiones de grupo. Otra psicóloga estudiante de $4 .^{\circ}$ de Grado en Psicología ayudaba en la toma de notas de la información verbal ofrecida por cada participante y en la realización de preguntas cuando lo consideraba pertinente.

\section{Análisis}

Para la realización del análisis estadístico se empleó el programa estadístico IBM SPSS 22.0 para Windows. En cuanto a los datos obtenidos mediante el focus group, las entrevistas fueron transcritas. Posteriormente, para su análisis cualitativo, se hizo una puesta en común de las contestaciones de los pacientes analizando las respuestas más frecuentes.

\section{Resultados}

Tabla 1

Puntuación media y desviación típica en la escala de dificultades en la regulación emocional (DERS) según subescalas y total

\begin{tabular}{lccc}
\hline & Media muestra control & Media & Desviación típica \\
\hline Desatención emocional & 9,8 & 10,92 & 3,37 \\
Confusión emocional & 7,6 & 10,42 & 4,10 \\
Rechazo emocional & 14,7 & 24,42 & 7,99 \\
Interferencia cotidiana & 9,7 & 12,42 & 4,81 \\
Descontrol emocional & 15,7 & 25,92 & 10,15 \\
Total desregulación & 57,4 & 84,08 & 23,77 \\
emocional & & & \\
\hline
\end{tabular}

En cuanto a la escala de dificultades en la regulación emocional (DERS), podemos decir que, en todas las subescalas, los pacientes muestran puntuaciones por encima de los puntos de corte establecidos de una muestra control (Hervás y Jodar, 2004), por lo que se aprecia que la desregulación emocional está presente en la muestra empleada de pacientes con diagnóstico de juego patológico. 
Tabla 2

Puntuación media y desviación típica en la escala de impulsividad de Barrat, (BIS-11) según subescalas y total

\begin{tabular}{lccc}
\hline & Media muestra control & Media & Desviación típica \\
\hline Impulsividad cognitiva & 16,8 & 11,83 & 4,22 \\
Impulsividad motora & 22,4 & 12,75 & 4,90 \\
Impulsividad no & 23,6 & 15,83 & 3,64 \\
planificada & $52-71$ & 40,42 & 10,31 \\
Total impulsividad & & & \\
\hline
\end{tabular}

Respecto a la escala de impulsividad de Barrat (BIS-11), encontramos que las puntuaciones de todas las subescalas, incluida la del constructo general, no se encuentran por encima de la media de la muestra control (Patton y Stanford, 1995), por lo que podemos considerar que no hay una presencia destacada de esta medida de impulsividad en la muestra del estudio, al menos evaluada mediante este instrumento.

Tabla 3

Respuestas más comunes en la sesión de focus group

«Juego para afrontar ciertas situaciones: problemas familiares, con la pareja, en el trabajo, deudas, recuperar pérdidas, etc.»

«Juego con más frecuencia...»

«Cuando me encuentro desanimado, enfadado, preocupado, rabioso, culpable...» (emociones negativas)

«Cuando me encuentro más animado, seguro de mí mismo o eufórico» (emociones positivas)

«Durante el juego...»

«Juego para que me disminuya o desaparezca dicho malestar generado por las emociones negativas»

«Esa emoción positiva se mantiene o incluso puede aumentar»

«Al finalizar la conducta de juego...»

EN CASO DE PERDER: «Me siento con un estado de ánimo más bajo, más triste, enfadado conmigo mismo, amargura, vergüenza, rabia, etc.»

EN CASO DE GANAR: «Eufórico, más contento, con más esperanzas, mi estado de ánimo sube, capacidad de logro para ganar etc.»

Acorde a la información cualitativa del focus group, es interesante resaltar la importancia de la influencia de las emociones en el inicio y el trascurso del juego patológico.

\section{Discusión y conclusiones}

Por lo que respecta a la importancia de la relación entre el juego y la creencia de lograr estados emocionales positivos o disminuir los estados emocionales negativos (Shead, Callan y Hodgins, 2008), se encontró que las puntuaciones, según escala de dificultades en la regulación emocional (DERS), se muestran por encima de la media, al compararlo con los puntos de corte 
de una muestra control. Los datos hallados han permitido poner de manifiesto, una vez más, la dificultad de la regulación emocional en los jugadores patológicos (Williams y cols., 2012).

Por otra parte, y en contra de lo encontrado habitualmente (véase Blanco y cols., 2009; Fernández y Echeburúa, 2006), las puntuaciones de impulsividad, según la escala de impulsividad de Barrat (BIS-11), no se encuentran por encima de los baremos de corte, al compararlo con las medias de una muestra control. Esto nos indica que no se aprecia una relación destacable entre la impulsividad y el juego patológico en la muestra del estudio, al menos evaluada con esta media.

Finalmente, cabe destacar, de acuerdo al focus group, la importancia del manejo de las emociones en el inicio y el trascurso del juego patológico (Korman y cols., 2008). Estos resultados nos sugieren una propuesta del entrenamiento en regulación emocional para la prevención e intervención eficaz en el tratamiento del juego patológico, como, por ejemplo, el entrenamiento de estrategias de autocontrol para manejar las emociones de manera adaptativa.

\section{Referencias bibliográficas}

American Psychiatric Association (2013). DSM-V. Diagnostic and statistical manual of mental disorders (5. ${ }^{a}$ edición). Arlington, VA: APA.

Becoña, E. (1993). El juego compulsivo en la comunidad autónoma gallega. Santiago de Compostela: Consellería de Sanidade da Xunta de Galicia.

Becoña, E. (2004). El juego patológico: Prevalencia en Espana. Salud y drogas, 4, 9-34.

Blanco, C., Potenza, M. N., Kim, S. W., Ibáñez, A., Zaninelli, R., Sáiz Ruiz, J. y Grant, J. E. (2009). A pilot study of impulsivity and compulsivity in pathological gambling. Psychiatry Research, 167, 161-168.

Bottesi, G. y Ghisi, M. (2014). Pathological gambling: Compulsive-impulsive spectrum disorder, behavioural addiction or both? Phenotypic and endophenotypic evidence. Psychopathology Review, 1, 2-25.

Caballo, V. E., Torres, I. C. S. e Isabel, J. A. C. (2011). Manual de psicopatología y trastornos psicológicos. Barcelona: Pirámide.

Candiani Garza, M. (2012). Jugar sin límites. Manual de ludopatía dirigido al jugador compulsivo y su familia. México: Font.

Chóliz, M. (2006). Adicción al juego de azar. Recuperado el 29 de abril de 2016 de: http://www. uv.es/choliz/ADICCION\%20AL\%20JUEGO\%20(MCholiz).pdf

Domínguez Álvarez, A. M. (2007 a). Introducción. En S. Moreno Mariscal (coord.), Guía clínica. Actuar ante el juego patológico (pp. 49-57). Sevilla: Consejería para la Igualdad y Bienestar Social de la Junta de Andalucía.

Domínguez Álvarez, A. M. (2007 b). Prevalencia de juego patológico. En S. Moreno Mariscal (coord.), Guía clínica. Actuar ante el juego patológico (pp. 49-57). Sevilla: Consejería para la Igualdad y Bienestar Social de la Junta de Andalucía.

Domínguez, A. (2009). Epidemiología y factores implicados en el juego patológico. Apuntes de psicología, 27, 3-20.

Estévez, A., Herrero, D., Sarabia, I. y Jauregui, P. (2014). El papel mediador de la regulación emocional entre el juego patológico, uso abusivo de internet y videojuegos y la sintomatología disfuncional en jóvenes y adolescentes. Adicciones, 26, 282-290.

Fernández-Montalvo, J. y Echeburúa, E. (2006). Juego patológico y trastornos de personalidad: Un estudio piloto con el MCMI-II. Psicothema, 18, 453-458. 
French, M. T., Maclean, J. C. y Ettner, S. L. (2008). Drinkers and bettors: Investigating the complementarity of alcohol consumption and problem gambling. Drug and alcohol dependence, 96, 155-164.

Freud, S. (1948). Obras completas, Madrid: Biblioteca Nueva.

Gratz, K. L. y Roemer, L. (2004). Multidimensional assessment of emotion regulation and dysregulation: Development, factor structure, and initial validation of the difficulties in emotion regulation scale. Journal of psychopathology and behavioral assessment, 26, 41-54.

Gerstein, D., Murphy, S., Toce, M., Hoffman, J., Palmer, A., Johnson, R. y Sinclair, S. (1999). Gambling impact and behavior study (Report to the National Gambling Impact Study Commission). National Opinion Research Center. Chicago, IL.

Griffiths, M. y Wood, R. T. (2000). Risk factors in adolescence: The case of gambling, videogame playing, and the Internet. Journal of gambling studies, 16, 199-225.

Jiménez-Murcia, S., Pérez, R. G., Fernández-Aranda, F., Moya, E. Á., Aymamí, M. N., GómezPeña, M. y cols. (2009). Comorbilidad del juego patológico: variables clínicas, personalidad y respuesta al tratamiento. Revista de Psiquiatría y Salud Mental, 2, 178-189.

Jiménez Rodríguez, J. y Fernández de Haro, E. (1999). Estudio de algunas variables sociodemográficas relevantes en jugadores patológicos que acuden a tratamiento. Análisis y Modificación de Conducta, 25, 51-80.

Korman, L. M., Collins, J., Dutton, D., Dhayananthan, B., Littman-Sharp, N. y Skinner, W. (2008). Problem gambling and intimate partner violence. Journal of Gambling Studies, 24, 1323.

Montero, I. y León, O. G. (2007). Guía para nombrar los estudios de investigación en Psicología. International Journal of Clinical and Health Psychology, 7, 847-862.

Odlaug, B. L., Schreiber, L. R. N. y Grant, J. E. (2013). Personality dimensions and disorders in pathological gambling. Current Opinion in Psychiatry, 26, 107-112.

Oquendo, M. A., Baca-García, E., Graver, R., Morales, M., Montalvan, V. y Mann, J. J. (2001). Spanish adaptation of the Barratt impulsiveness scale (BIS-11). The European journal of psychiatry, 15, 147-155.

Shead, N., Callan, M. J. y Hodgins, D. C. (2008). Probability discounting among gamblers: Differences across problem gambling severity and affect-regulation expectancies. Personality and Individual Differences, 45, 536-541.

Wardle, H., Moody, A., Griffiths, M., Orford, J. y Volberg, R. (2011). Defining the online gambler and patterns of behaviour integration: Evidence from the British Gambling Prevalence Survey 2010. International Gambling Studies, 11, 339-356.

Williams, A. D., Grisham, J. R., Erskine, A. y Cassedy, E. (2012). Deficits in emotion regulation associated with pathological gambling. British Journal of Clinical Psychology, 51, 223238.

Williams, R. J., Volberg, R. A. y Stevens, R. M. (2012). The population prevalence of problem gambling: Methodological influences, standardized rates, jurisdictional differences, and worldwide trends. Ontario Problem Gambling Research Centre. 